\title{
Route to shrimps: dissipation driven formation of shrimp-shaped domains
}

\author{
Roxána Varga ${ }^{\mathrm{a}}$, Kálmán Klapcsik ${ }^{\mathrm{a}}$, Ferenc Hegedús ${ }^{\mathrm{a}}$ \\ ${ }^{a}$ Department of Hydrodynamic Systems \\ Faculty of Mechanical Engineering \\ Budapest University of Technology and Economics
}

\begin{abstract}
In this paper, two scenarios for the formation of shrimp-shaped domains [1] are presented. The employed test model is the Keller-Miksis equation that is a second order, harmonically forced nonlinear oscillator describing the dynamics of a single spherical gas bubble placed in a liquid domain. The results have shown that with an increasing dissipation rate (liquid viscosity), shrimp-shaped domains are evolved within the complex structure of each subharmonic resonances in the amplitude-frequency parameter plane of the external forcing. The mechanism is the coalescence and interaction of two pairs of a period-doubling and a saddle-node codimension-two bifurcation curves.

Keywords: shrimp-shaped domains, GPU programming, bifurcation structure, high dissipation rate, Keller-Miksis equation, bubble dynamics
\end{abstract}

\section{Introduction}

The rapidly increasing computational capacities have opened the way to examine bifurcation structures of various systems in many fields of sciences in multi-dimensional parameter space [2, 3, 4, 5, 6, 7, 8, 9, 10]. For example, based on the shooting method, 5 several high-resolution studies have revealed the existence of shrimp-shaped domains (SSD) in bi-parametric planes [11, 12, 13, 14, 15, 16, 17, 18, 19, 20, 21]. They are a special class of codimension-two isoperiodic stable structures (ISS) [18], and they turned out to be an efficient "tool" to handle multi-stability [22] and to control chaos

Email address: rvarga@hds.bme.hu (Roxána Varga) 
[21, 23].

The bifurcation structure of a typical SSD is presented in Fig.1 in a two-dimensional parameter plane (the physical meaning of the parameters are unimportant here). In the upper panel, the periods of the found attractors are shown up to period- 6 computed by an initial value problem solver. In the bottom panel, the skeleton of the underlying SSD is presented via co-dimension two bifurcation curves computed by a boundary value problem solver. The solid and dashed curves are saddle-node (SN) and period doubling (PD) bifurcations, respectively. The skeleton of an SSD can always be characterised as follows. It consists of a U-shaped SN curve bounding the SSD from one side, an SN curve inside having a co-dimension two cusp bifurcation and two crossing PD curves. Observe that the two sides of the PD bifurcations approach to different SN curves. The formation of such two-dimensional SSD structures are in the main focus of the present study. For a detailed categorisation of their structure, see also paper [14].

The presence of shrimps were first identified in a discrete-time system [11]; namely, in the Hénon-map. Bonatto et. al [1] successfully found these domains also in a continuous-time $\mathrm{CO}_{2}$ laser system, studied also experimentally by Arecchi et. al [24]. Later, the existence of SSDs in different systems were reported [12]; and revealed their organization along straight lines [14, 19], around spiral hubs [25, 26] or as zig-zag patterns [27] indicating the extremely high complexity of the underlying bifurcation structure.

The majority of the studies in the literature use solely the shooting method via employing a suitable initial value problem solver. The shrimp-shaped domains, however, usually emerge inside large chaotic domains (this is the main reason they are also called isoperiodic stable structures), and in case of multi-stability and without the proper filtering of the results, they can be "buried" by the co-existing chaos [22]. Another way to properly identify SSDs is to use a numerical continuation technique [28], and trace 35 the bifurcation curves composing the skeleton of the shrimps [18, 29].

With such an approach, Klapcsik et. al [22] successfully explored a complete family of period-3 SSDs organized in a zig-zag pattern in the amplitude-frequency parameter plane of the external forcing of their model. They employed the harmonically driven Keller-Miksis equation [30, 31] during their computations, which is a second 

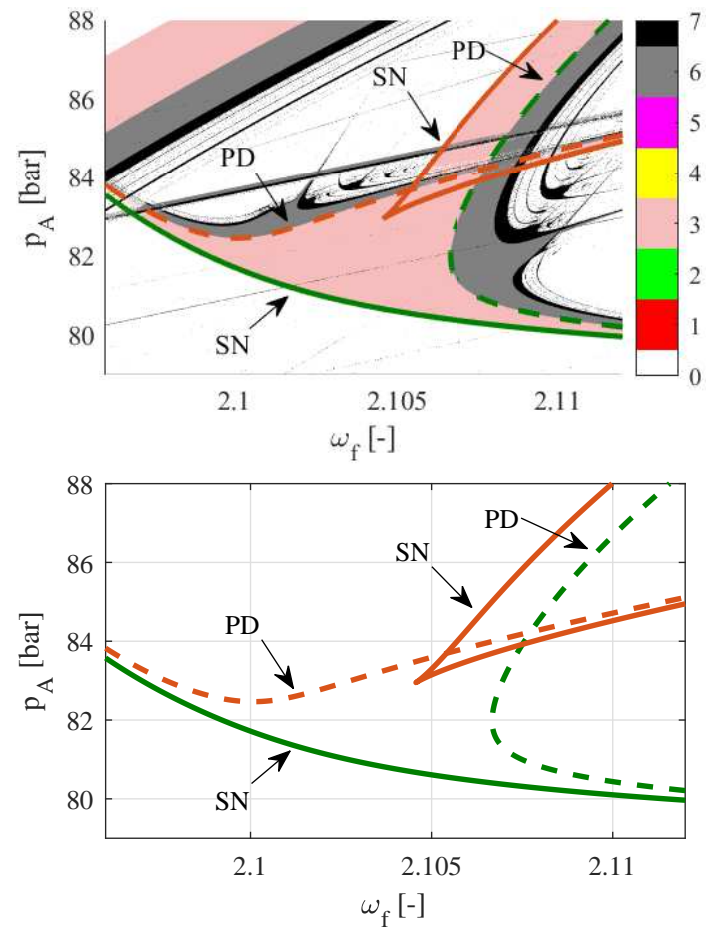

Figure 1: The bifurcation structure of a typical shrimp-shaped domain (SSD). Upper panel: periodicity diagram with periods up to period-6. Lower panel: the skeleton of the SSD composed by saddle-node (SN) and period doubling (PD) bi-parametric bifurcation curves.

order ordinary differential equation describing the radial dynamics of a single spherical gas bubble placed in a liquid domain [6, 32, 33, 34, 35, 36, 37, 38, 39, 40, 41]. The special property of their system was the very strong dissipation rate originated from the high viscosity of the liquid domain (glycerine). Interestingly, in our preliminary results obtained in water having orders of magnitude smaller damping rate, employing 45 the same mathematical model, the above mentioned SSDs are absent. Consequently, the dissipation rate must play a significant role in the formation of SSDs at least in case of the Keller-Miksis nonlinear oscillator.

The main objective of the present study is to reveal the possible mechanisms of the formation of SSDs by systematically increasing the viscosity (dissipation rate of the system) of the used liquid (initially water) via altering the composition of a waterglycerine mixture. We found that the skeletons of the studied SSDs in our model 
are always formed via the interaction of two pairs of a period doubling (PD) and a saddle-node (SN) codimension-two bifurcation curves. The structure of these skeletons and their corresponding bifurcation curves are described via the well-known winding number [2, 8, 42] (order of the curves) and compared with the results already presented in the literature. In order to successfully achieve the aforementioned goal, the shooting method and the continuation technique were combined.

\section{The mathematical model}

The bubble oscillator used for the computations in this paper is the slightly modified form [31] of the Keller-Miksis equation [30]:

$$
\begin{aligned}
\left(1-\frac{\dot{R}}{c_{L}}\right) R \ddot{R}+\left(1-\frac{\dot{R}}{3 c_{L}}\right) & \frac{3}{2} \dot{R}^{2}= \\
& \frac{1}{\rho_{L}}\left(1+\frac{\dot{R}}{c_{L}}\right)\left(p_{L}-p_{\infty}(t)\right)+\frac{R}{\rho_{L} c_{L}} \frac{d\left(p_{L}-p_{\infty}(t)\right)}{d t} .
\end{aligned}
$$

This nonlinear, second order ordinary differential equation describes the time evolution

of the radius $R(t)$ of a spherical bubble. It takes into account the compressibility of the liquid domain to the first order; thus, it incorporates sound radiation. The dot stands for the derivative with respect to time. In Eq. (1), $\rho_{L}$ and $c_{L}$ are the density and the sound speed of the liquid, respectively.

The pressure far away from the bubble is

$$
p_{\infty}=P_{\infty}+p_{A} \sin (\omega t)
$$

where $P_{\infty}$ is the static or ambient pressure, $p_{A}$ is the pressure amplitude and $\omega$ is the angular frequency of the periodic excitation. The mechanical equilibrium at the bubble wall can be written as

$$
p_{G}+p_{V}=p_{L}+\frac{2 \sigma}{R}+4 \mu_{L} \frac{\dot{R}}{R},
$$

where the total pressure inside the bubble is the sum of the partial pressures of the noncondensable gas content $p_{G}$ and the vapour pressure $p_{V}$. The pressure at the bubble wall in the liquid side is $p_{L}$. The surface tension is $\sigma$ and the liquid dynamic viscosity is $\mu_{L}$. The gas inside the bubble obeys a simple polytropic relationship:

$$
p_{G}=\left(\frac{2 \sigma}{R_{E}}-p_{V}+P_{\infty}\right)\left(\frac{R_{E}}{R}\right)^{3 n},
$$


where $R_{E}$ is the equilibrium radius (size of the bubble) and $n$ is the polytropic exponent.

\subsection{The dimensionless equation system}

For the numerical simulations, Eqs. (1)-(4) need to be rewritten into a first order dimensionless differential equation system. By the introduction of the dimensionless time $\tau=t \omega /(2 \pi)$, the dimensionless bubble radius $y_{1}=R / R_{E}$ and the bubble wall velocity $y_{2}=2 \pi \dot{R} /\left(R_{E} \omega\right)$, the system can be written as

$$
\begin{aligned}
& y_{1}^{\prime}=y_{2}, \\
& y_{2}^{\prime}=\frac{N}{D},
\end{aligned}
$$

where ' denotes the derivative with respect to $\tau$. After some algebraic manipulation of the model, the numerator $N$ can be defined as

$$
\begin{gathered}
N=\left(C_{1}+C_{2} y_{2}\right)\left(\frac{1}{y_{1}}\right)^{C_{9}}-C_{3}\left(1+C_{8} y_{2}\right)-\left(C_{4}+C_{5} y_{2}\right) \frac{1}{y_{1}} \\
-C_{6}\left(1+C_{8} y_{2}\right) \sin (2 \pi \tau)-1.5\left(1-\frac{1}{3} C_{8} y_{2}\right) y_{2}^{2}-C_{7} \cos (2 \pi \tau) y_{1}
\end{gathered}
$$

and the denominator $D$ as

$$
D=y_{1}-C_{8} y_{2} y_{1}+C_{5} C_{8} .
$$

The parameters in Eqs. (6) and (7) are defined as follows

$$
\begin{aligned}
C_{1} & =\left(\frac{2 \sigma}{R_{E}}-p_{V}+P_{\infty}\right) /\left(\rho_{L} R_{E}^{2}\left(\frac{\omega}{2 \pi}\right)^{2}\right) \\
C_{2} & =\left(\frac{2 \sigma}{R_{E}}-p_{V}+P_{\infty}\right)(1-3 n) /\left(c_{L} \rho_{L} R_{E} \frac{\omega}{2 \pi}\right) \\
C_{3} & =\left(p_{V}-P_{\infty}\right) /\left(\rho_{L} R_{E}^{2}\left(\frac{\omega}{2 \pi}\right)^{2}\right) \\
C_{4} & =\frac{2 \sigma}{R_{E}} \rho_{L} R_{E}^{2}\left(\frac{\omega}{2 \pi}\right)^{2} \\
C_{5} & =\left(4 \mu_{L}\right) /\left(\rho_{L} R_{E}^{2} \frac{\omega}{2 \pi}\right) \\
C_{6} & =p_{A} /\left(\rho_{L} R_{E}^{2}\left(\frac{\omega}{2 \pi}\right)^{2}\right) \\
C_{7} & =p_{A} /\left(c_{L} \rho_{L} R_{E} \frac{\omega}{(2 \pi)^{2}}\right) \\
C_{8} & =\left(R_{E} \omega\right) /\left(2 \pi c_{L}\right) \\
C_{9} & =3 n .
\end{aligned}
$$


Since the angular frequency can vary on a scale of many orders of magnitude, it is reasonable to normalize it with a suitable reference quantity. Therefore, a dimensionless relative frequency was used for the computations defined as

$$
\omega_{f}=\frac{\omega}{\omega_{0}},
$$

where $\omega_{0}$ is the linear resonant frequency of the system. According to Brennen [33], $\omega_{0}$ can be calculated from the following equation:

$$
\omega_{0}=\sqrt{\frac{3 n\left(P_{\infty}-p_{V}\right)}{\rho_{L} R_{E}^{2}}+\frac{2(3 n-1) \sigma}{\rho_{L} R_{E}^{3}}-\frac{4 \mu_{L}^{2}}{\rho_{L}^{2} R_{E}^{4}}} .
$$

\subsection{Control parameters and material properties of the system}

The objective of the present study is to track the evolution of the shrimp-shaped domains in the parameter plane $p_{A}-\omega_{f}$ of the harmonic driving with increasing dissipation rate. Accordingly, the two main control parameters are the pressure amplitude $p_{A}$ and the relative frequency $\omega_{f}$ of the system. Their values are within the limits of $0-100$ bar and $1-14$, respectively. Observe that most of the applied relative frequency values are well above the main resonance frequency $\omega_{f}=1$; thus, subharmonic resonances shall dominate the investigated parameter plane.

The low viscosity (low dissipation rate) reference computation was carried out using pure water at constant ambient pressure $P_{\infty}=1$ bar and at constant ambient temperature $T_{\infty}=25^{\circ} \mathrm{C}$. These ambient quantities specify all the liquid material properties determined by means of the Haar-Gallagher-Kell equation of state [43]. The calculated values for this reference case are listed in Table1, In order to reveal the effect of the dissipation rate, computations were repeated at several values of the liquid viscosity summarized in Table 2 These values were chosen according to the volume fractions of water glycerin mixtures. However, from the material properties, only the viscosity was changed to clearly separate the effect of the dissipation. During the computations, the equilibrium bubble radius (bubble size) was $R_{E}=0.1 \mathrm{~mm}$ and the polytropic exponent was $n=1.4$ assuming adiabatic state of change and diatomic molecules for the gas content. 
Table 1: Liquid properties for pure water calculated at $T_{\infty}=25^{\circ} \mathrm{C}$ and at $P_{\infty}=1$ bar ambient properties by means of the Haar-Gallagher-Kell equation of state [43].

\begin{tabular}{ll}
\hline material property & value \\
\hline$p_{V}$ & $3166.8 \mathrm{~Pa}$ \\
$\sigma$ & $0.0720 \mathrm{~N} / \mathrm{m}$ \\
$\mu_{L}$ & $0.00089 \mathrm{~Pa} \mathrm{~s}$ \\
$\rho_{L}$ & $997.064 \mathrm{~kg} / \mathrm{m}^{3}$ \\
\hline
\end{tabular}

Table 2: Values of the liquid dynamic viscosities used during the computations, calculated via the volume fraction of the species. [44]

\begin{tabular}{lll}
\hline water $(L)$ & glycerine $(L)$ & $\mu_{L}[\mathrm{Pas}]$ \\
\hline 1 & 0 & 0.00089 (pure water) \\
1 & 0.8 & 0.00506 \\
1 & 1.3 & 0.0102 \\
1 & 3.35 & 0.0498 \\
1 & 5.2 & 0.100 \\
1 & 10.7 & 0.252 \\
1 & 26 & 0.501 \\
\hline
\end{tabular}




\section{The exoskeleton of the subharmonic resonances for pure water}

Before the examination of SSDs - similarly to the study of Klapcsik et al. [22] - the present investigation also starts with the exploration of subharmonic resonances Throughout this section, computations are performed only for the low viscosity reference case (pure water, Table 1). Although the employed model is the same as in [22], the more than three orders of magnitude difference in the liquid viscosity (approximately $\times 320$ ) shall result in a structural difference in the subharmonic bifurcation set.

To quickly obtain a picture about the periodic solutions, Sys. (5) is first solved as an initial value problem (IVP) applying 25 randomized initial conditions at every parameter combinations to reveal the co-existing stable states (attractors). The numerical scheme is the explicit and adaptive Runge-Kutta-Cash-Karp method with embedded error estimation of orders 4 and 5, the algorithm is adapted from [45]. Both the absolute and relative errors are $10^{-10}$. The data for the first 2048 iterations are regarded as initial transients and discarded. One iteration means the integration of the system forward in time by one cycle of the excitation. After the initial transients, several characteristic properties of the found attractors are recorded: points of their Poincaré section $P\left(y_{1}\right)$ and $P\left(y_{2}\right)$, their period $m$ and their torsion number $n$. The torsion number $n$ is the average number of rotations of a neighbouring orbit around a given solution during one of its period [42, 46, 47]; and it is an integer number near a bifurcation point. Therefore, the order of a bifurcation point can be defined via the pair of its torsion number and its period $(n, m)$, for details see [48]. According to several studies in the literature [8], the order is turned out to be a very efficient tool for the description of bifurcation structures demonstrated in the present study as well.

Out of the several computations, Fig. 2 2 shows only one typical bifurcation structure of the stable periodic solutions with the pressure amplitude of the excitation $p_{A}$ as control parameter at constant relative frequency $\omega_{f}=5$. Here, the first coordinate of the Poincare section $P\left(y_{1}\right)$ of the converged solutions is plotted versus the control parameter varied between 0 and 25 bar with 0.01 bar increment. The present study focuses only on the structure of periodic orbits; thus, the chaotic solutions are filtered 
from this figure. All of the periodic attractors emerge via a saddle-node $S N$ bifurcation except the period-1 orbit, which is originated from the equilibrium solution $P\left(y_{1}\right)=1$ at $p_{A}=0$. After some increase of the control parameter, these solutions go through period-doubling cascades and finally they lose their stability.

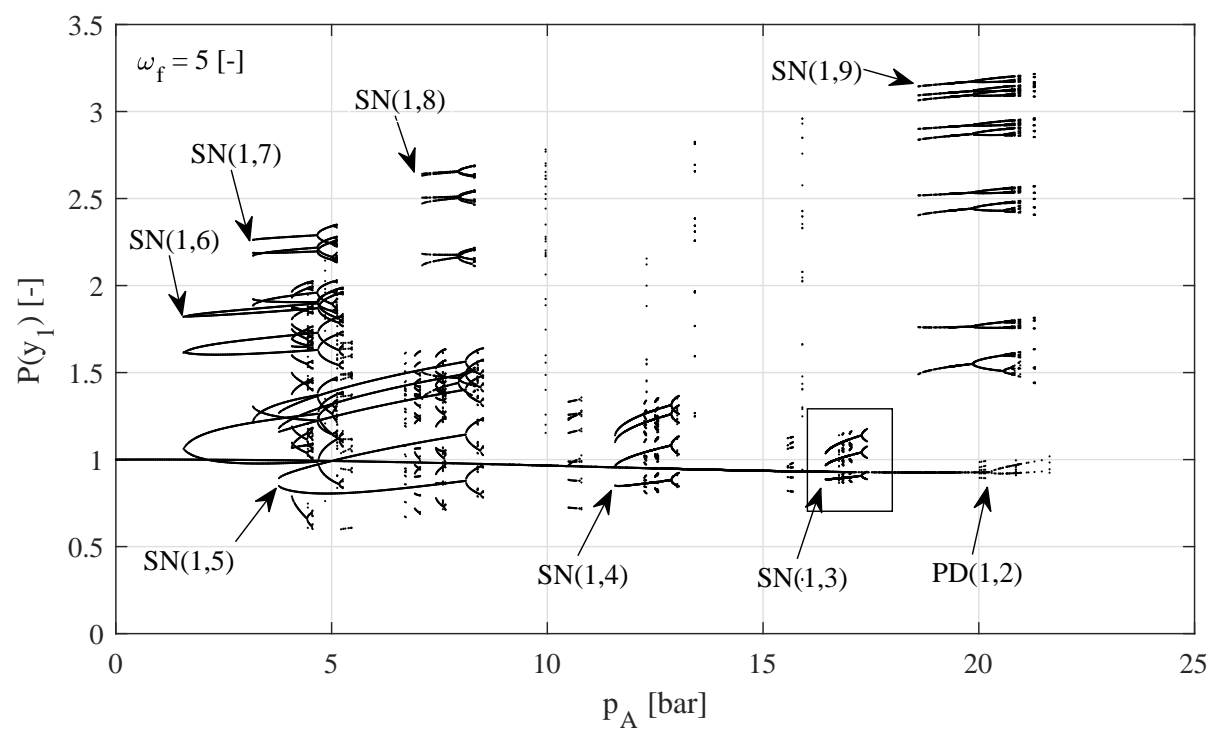

Figure 2: Bifurcation diagram of the stable periodic solutions at $\omega_{f}=5$, where the first component of the Poincaré section $P\left(y_{1}\right)$ is plotted as a function of the pressure amplitude $p_{A}$. The arrows marking the $S N$ and $P D$ points are subjects of further study. The order of these bifurcation points are marked by $S N(n, m)$ and $P D(n, m)$, respectively. The box highlights the region where the $\mathrm{SN}(1,3)$ solution exists. Chaotic orbits are omitted from the figure.

The orbits which are the subject of further study are marked by their order $S N(n, m)$ or $P D(n, m)$, see the arrows pointing to the corresponding bifurcation points. Observe that the torsion numbers $n$ of these points are always 1; that is, they all belong to the family of subharmonic resonances of order $(1, m)$, where the period $m$ is between 1 and

9. These subharmonics also exist in the bifurcation structure of Klapcsik et al. [22], in spite of the applied high dissipation rate there. The investigation of the higher order subharmonics $(n>1)$ are beyond the scope of the present paper. These subharmonics appear in Fig. 2 as solutions with very high periods. Interestingly, they are completely absent in the bifurcation structures of Klapcsik et al. [22]; that is, only such higher 
order subharmonics are damped out with increasing liquid viscosity.

In order to obtain a global overview of the subharmonic resonances in the excitation parameter plane, the bifurcation points marked in Fig.2 and their first period-doubling points in the Feigenbaum cascade are tracked down as a function of the pressure amplitude $p_{A}$ and relative frequency $\omega_{f}$. Altogether $8 \times 2=16$ bifurcation points are tracked. This task can be easily done by reformulating the problem into a boundary value problem (BVP). For a period $m$ solution, the periodic boundary condition is defined as

$$
\underline{y}(0)=\underline{y}\left(m \tau_{0}\right),
$$

where $m$ is the period of the desired solution and $\tau_{0}=1$ is the dimensionless period of the excitation. For these calculations, the AUTO continuation and bifurcation analysis software was used [28]. For a detailed study on the capabilities of this software, the reader is kindly referred to the publications [32, 49, 50, 51, 52].

The resulted codimension-two bifurcation curves are summarized in Fig. 3. The solid and dashed curves are related to the $S N$ and $P D$ bifurcation points, respectively. The vertical red line at $\omega_{f}=5$ shows the parameter range corresponding to Fig.2. In Fig. 3, a remarkable U-shaped ordering of the subharmonic structure arise, which was hidden in the one-dimensional cut shown in Fig.2 The same U-shaped structures characterize the subharmonics also in case of high dissipation, see again Ref. [22]. The main aim of the following sections is to highlight the differences between the internal structures of a subharmonic resonance corresponding to high and low liquid viscosities, and to describe the formation of SSDs.

\section{The internal structure of the period-3 subharmonic resonance}

With a BVP solver, both stable and unstable solutions can be easily computed. Moreover, along a traced bifurcation curve in one-dimension, bifurcation points can also be detected. Therefore, the BVP solver AUTO used for our computations is a perfect tool to explore the internal structure of a subharmonic resonance. The strategy is to calculate several one-dimensional fixed-point curves [53, 54] as a function only 


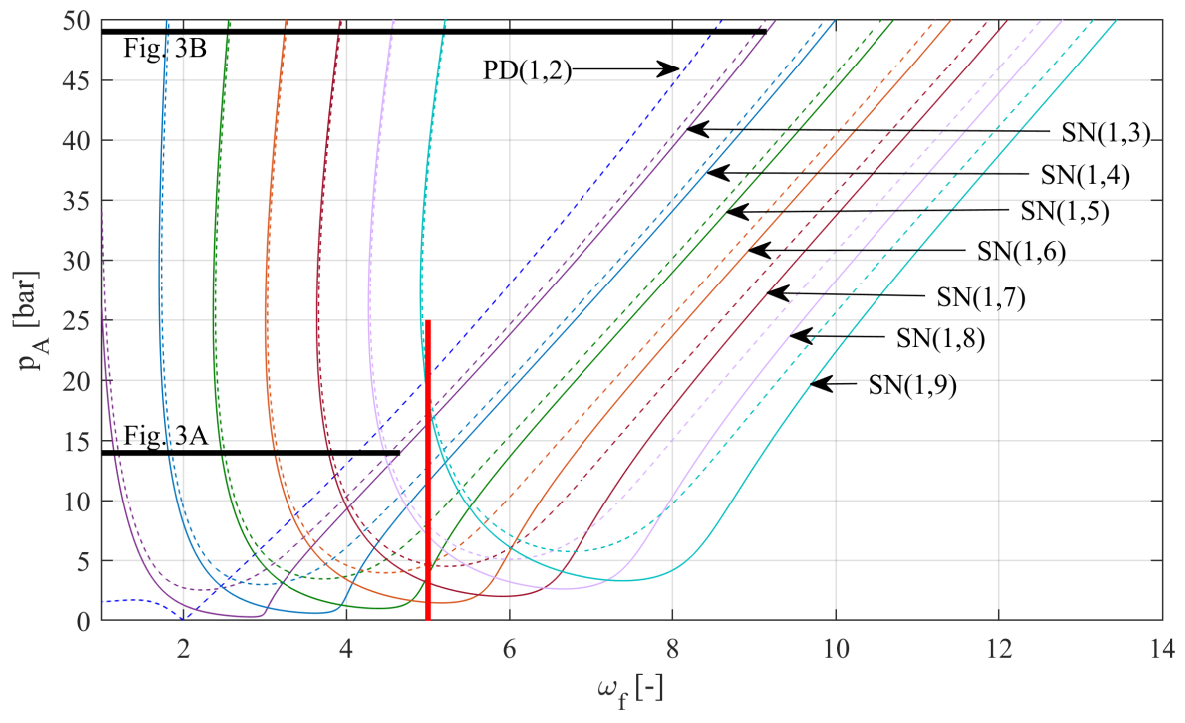

Figure 3: Codimension-two bifurcation curves corresponding to the marked bifurcation points in Fig. 2 and to their subsequent period-doubling points. The solid and dashed lines are the saddle-node $S N$ and the period-doubling $P D$ curves, respectively. The red vertical line denotes the parameter range of the computations presented in Fig.2 The lower and upper black horizontal lines denote the parameter ranges of the computations presented in Fig. 4.4 and B, respectively.

subharmonic resonance curve of order $S N(1,3)$ (see again Fig. 3). The next step is the computation of all the codimension-two curves (again in the $p_{A}-\omega_{f}$ plane) initiated from the detected $S N$ and $P D$ points in the one-dimensional $\omega_{f}$-sections. With these steps, the whole internal structure of a subharmonic resonance can be explored.

Figure 4 presents two examples for frequency response curves ( $\omega_{f}$-sections) corresponding to the period-3 subharmonic resonance at pressure amplitudes $p_{A}=14 \mathrm{bar}$ (panel A) and at $p_{A}=49 \mathrm{bar}$ (panel B). Here the maximum value of the $y_{2}$ coordinate of the solution $\left(y_{2}^{\max }\right)$ is plotted versus the control parameter $\omega_{f}$. They were calculated as a function of the relative frequency $\omega_{f}$ at fixed pressure amplitudes initiated from the $\mathrm{SN}(1,3)$ curve (purple solid curve in Fig. 3). See also the two black horizontal lines in Fig. 3 at $p_{A}=14 \mathrm{bar}$ and at $p_{A}=49 \mathrm{bar}$, where these two $\omega_{f}$-section curves lie in the $p_{A}-\omega_{f}$ plane. In both Fig. $4 \mathrm{~A}$ ) and B), the red dashed lines mean unstable solutions, while the black solid segments are the stable orbits. The detected $P D$ and $S N$ 
points are marked by the black crosses and dots, respectively. The arrows point at these detected bifurcation points indicating their order $(n, m)$. For a better visualization, in panel B), only some segments of the curve are shown, and only some of the detected SN points are highlighted by arrows. The presented solutions have the following main features. First, the fixed-point curves at a given pressure amplitude are composed by closed loops. Along them, the bifurcation points appear as $S N-S N$ and $P D-P D$ pairs sequentially. Second, the regions of the existence of stable segments are really narrow because the $S N$ points are always very close to a $P D$ point; therefore, unstable orbits dominate the whole parameter domain, see also the magnification in panel A). This means that IVP solvers are really inefficient to obtain a good impression about such an internal structure. Third, although it is hard to visualize, but with increasing pressure amplitude, the number of the loops in the bifurcation curves are gradually increasing. At $p_{A}=14 \mathrm{bar}$, the number of the $S N-S N$ and $P D-P D$ pairs (and also the number of the loops) is 6 that increases to 16 at $p_{A}=49$ bar.
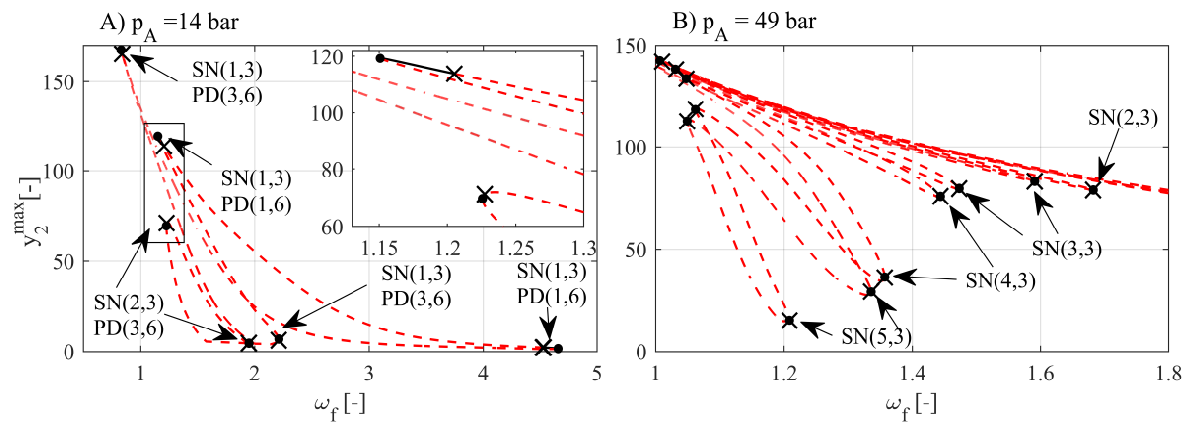

Figure 4: One-dimensional $\omega_{f}$ fixed-point diagrams corresponding to the subharmonic resonance of order $S N(1,3)$ at pressure amplitudes $p_{A}=14 \mathrm{bar}$ (panel A) and $p_{A}=49 \mathrm{bar}$ (panel B). $y_{2}^{\max }$ is the maximum value of the $y_{2}$ coordinate of the solution. The red dashed lines are the unstable solutions, while the black solid segments represent stable orbits. The $P D$ and $S N$ bifurcation points are marked by the black crosses and dots, respectively. The subfigure in panel A) shows the enlargement of the framed part of the fixed-point curve.

The internal structure of the period-3 subharmonic resonance can be revealed by following all the detected $S N$ and $P D$ bifurcation pairs of the $\omega_{f}$-sections in the biparametric plane of the pressure amplitude $p_{A}$ and the relative frequency $\omega_{f}$. The top 
panel of Fig. 5 shows this complex structure. Here, the continuous and dashed curves correspond to $S N$ and $P D$ bifurcations, respectively. The solid purple curve is the $\mathrm{SN}(1,3)$ resonance curve, and the dashed one is its corresponding PD curve, compare also with Fig. 3 The black horizontal lines with black crosses ( $P D$ points) and dots ( $S N$ points) are the sections of the frequency response curves shown in Fig 4 Since in Fig4 the consecutive $S N$ and $P D$ points are always very near to each other, in Fig.5 top, the black dots and crosses almost coincide. Observe how this internal structure incorporates an increasing number of codimension-two curves with increasing pressure amplitude. This reflects the increased number of $S N$ and $P D$ points found in Fig. $4 \mathrm{~B}$ compared to Fig. $4 \mathrm{~A}$. Observe also that the bifurcation curves can always be paired; that is, there is always a $P D$ curve moving close to an $S N$ curve, even if they are seemingly a single curve. To show this, the curve segments in the two rectangles in the top panel of Fig.5 are enlarged in Fig.6, in which the arrows again point at the curves indicating their bifurcation type and order. The alternating green-orange coloring serves only to distinguish these $S N-P D$ pairs of bifurcation curves more easily.

The description of the one-dimensional bifurcation structures, such as shown in Fig. 4. was discussed in great details in terms of the order of the bifurcation points in our previous paper, see again Klapcsik et al. [22]. Although that paper is related to a highly viscous liquid, the description is also valid for the present, low viscosity case (pure water). Thus, for the details, the interested reader is referred to the aforementioned publication. Despite of this similarity, the pattern of the corresponding internal resonance structure shows important differences. The zig-zag pattern clearly visible in Fig. 5 of Ref. [22] is totally absent in the internal structure shown in in the top panel of Fig. 5 of the present study.

Although the effect of the viscosity on the bifurcation structure shall be discussed in Sec.5 5 in details, in the bottom panel of Fig. 5 the internal structure of the subharmonic resonance of order $S N(1,3)$ for a much higher viscosity is already included here to introduce the term zig-zag pattern properly. Comparing the structures of the bifurcation curves of the two panels, one can clearly see that blocks a) and b) (discussed below) have no connections at low viscosity (pure water, upper panel), but for high enough viscosity (e.g. bottom panel), blocks a) and b) are connected in an alternating manner. 

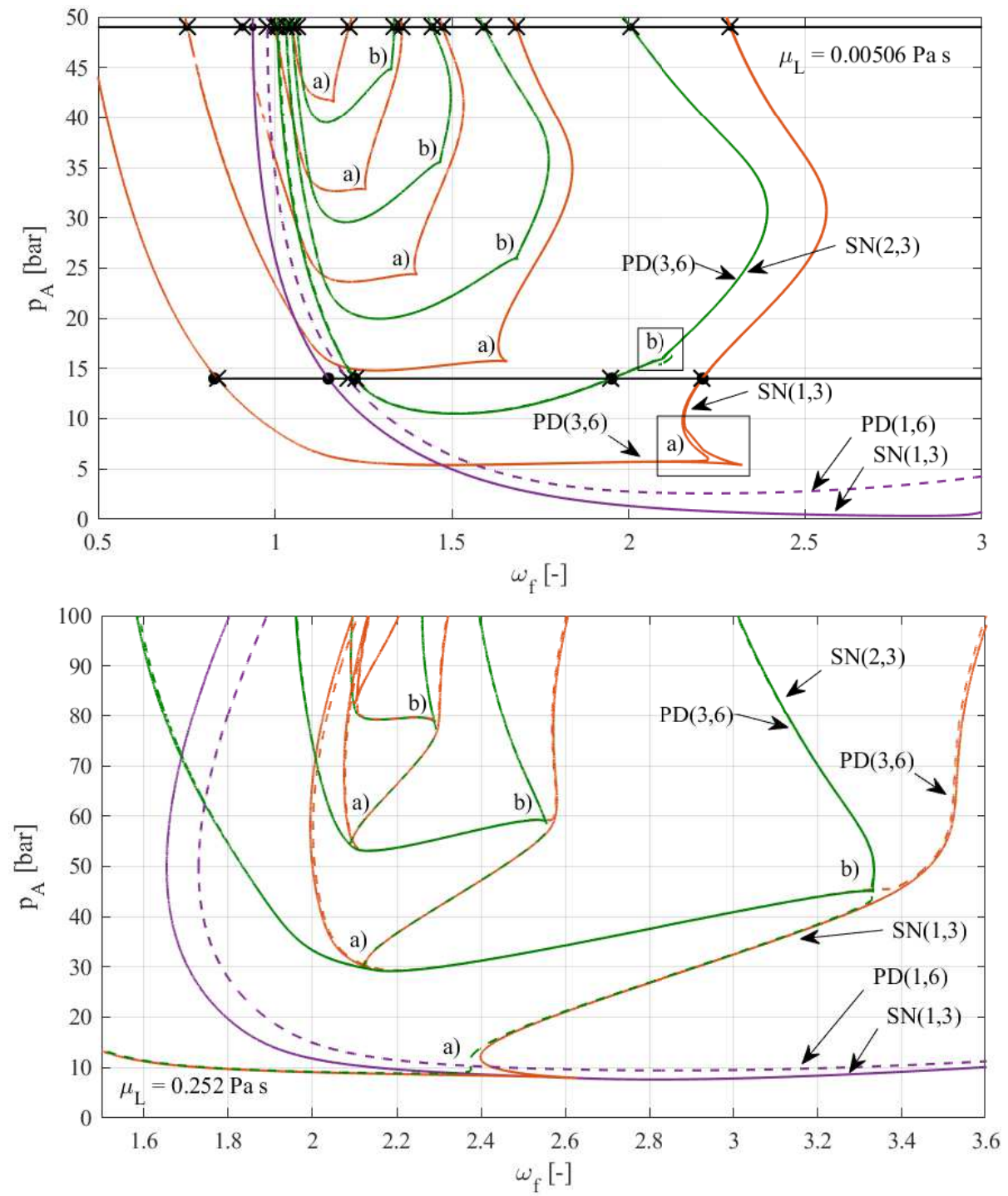

Figure 5: Top panel: The internal structure of the subharmonic resonance family of order $S N(1,3)$ (purple solid line, see also 3 at low viscosity (pure water). The horizontal black lines represent the parameter regions of the bifurcation curves presented in Fig. 44 A-B. The areas in the two rectangles are enlarged in Fig.6 The arrows show the type and order of the first few bifurcation curves. Bottom panel: the same internal structure of the subharmonic resonance family of order $\operatorname{SN}(1,3)$ at orders of magnitude higher viscosity. The zig-zag pattern of the bifurcation structure is clearly visible in this case. 


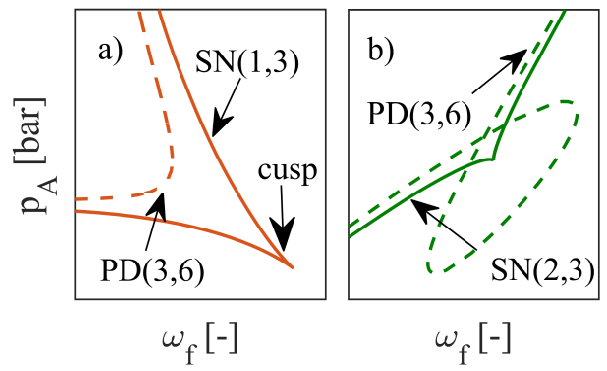

Figure 6: Magnifications of the bifurcation blocks of the predecessors of the shrimp-shaped domains (SSDs) marked by a) and b) in Fig.5 The arrows show the type and order of the curves they point to, compare with Fig. 5

Such an alteration is called the zig-zag pattern.

Since the "nodes" of the zig-zag blocks were turned out to be the skeleton of the shrimp-shaped domains (SSDs), see again Ref. [22], the viscosity of the liquid (dissipation rate) has to play an important role in their formation. In the next section, this scenario, the "rout to shrimps" shall be discussed in details by varying the liquid viscosity and monitor the evolution of the codimension-two curves presented in Fig.5.

Although in the top panel of Fig.5 the skeletons of SSDs are still missing, their "predecessors" are already visible shown by the block patterns a) and b). Two magnified examples are presented in Fig.6 for each cases. Both blocks consist of an $S N$ curve having a cusp bifurcation, and a parallel U-shaped (block a) or a looped (block b) $P D$ curve. These blocks, according to their patterns, are also marked by $a$ ) and $b$ ) in the top panel of Fig. 5 where they appear in an alternating order as the bifurcation curves emerge with increasing pressure amplitude $p_{A}$.

\section{The effect of the viscosity on the internal structure of the period-3 subharmonic resonance}

To reveal the possible routes to the shrimp-shaped domains via the formation of the zig-zag pattern, the calculations presented in the previous subsection are repeated at different dynamic viscosities $\mu_{L}$. For the used values of $\mu_{L}$, see again Table 2 Al-

though we only show the bi-parametric curves in the $p_{A}-\omega_{f}$ plane of the subharmonic 
resonance family of order $S N(1,3)$, the conclusions drawn are also valid for other subharmonic resonances based on our preliminary calculations. The results of the computations are summarized in Fig.7 The color coding of the curves is the same as in case of Fig. 5
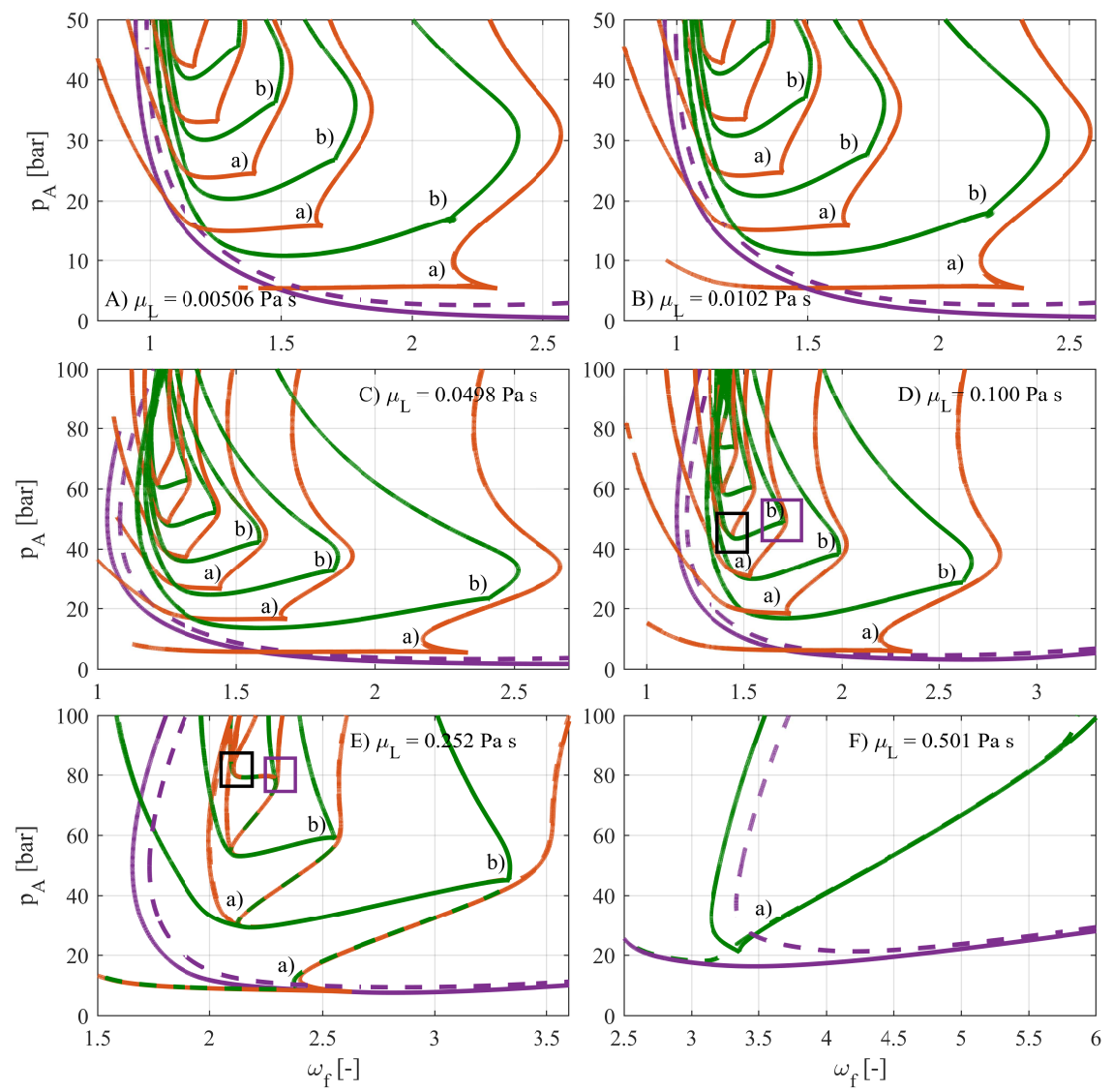

Figure 7: The codimension-two bifurcation curves of the subharmonic resonance of order $S N(1,3)$ (purple solid line) at different dynamic viscosities. The color code of the curves is the same as in case of Fig. 5 The bifurcation curves in the black and purple rectangles are magnified in Figs. 8 and 9 respectively.

With increasing dynamic viscosity $\mu_{L}$, two main alterations in the bifurcation structure can be recognized. Firstly, all the curves are shifted towards the higher pressure amplitudes. This is the reason of the extended parameter range of $p_{A}$ in Fig.7C-F. Observe that in panel F) only a few curves are left. In this case, the bifurcation curves are 
shifted upward significantly, and only a single block a) is visible. The system was in-

vestigated "only" up to pressure amplitude $p_{A}=100$ bar; thus, it is possible that block patterns a) and b) are gradually disappear with increasing viscosity. However, such an investigation is beyond the scope of the present paper. Since the main objective is the formation of SSDs with an increasing dissipation rate that can be clearly recognisable in the forthcoming discussions. Secondly, the block patterns a) and b) gradually approach to and collide with another pair of bifurcation curves initiating a complex interaction between two $S N-P D$ pairs, examined in details in Sec. 6. As the viscosity increases, this phenomenon takes place first with block patterns presented at high pressure amplitudes, and gradually spreads towards the lower amplitude regions forming the zig-zag pattern already observed in [22]. Compare also the upper panel (absence of zig-zag pattern) with the lower panel (presence of zig-zag pattern) in Fig.5, which are also the subfigures A) and E) in Fig.7.
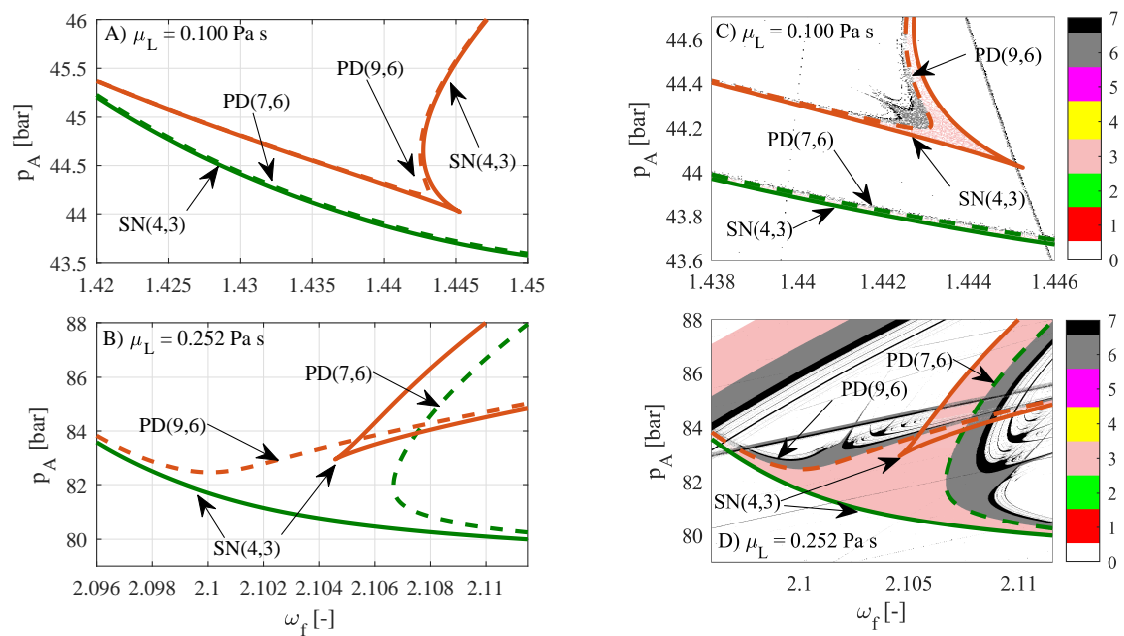

Figure 8: Resonance curves of block pattern a) creating a shrimp-shaped region as the liquid dynamic viscosity increases from $\mu_{L}=0.100 \mathrm{Pas}$ (top row) to $\mu_{L}=0.252 \mathrm{Pas}$ (bottom row). Panel A) and B) are the magnifications of the black rectangles in Fig.7P and E, respectively. Panel C) and D) are the results of an IVP scan of the parameter areas of panel A) and B), respectively. The color code means the period of the converged periodic solutions up to period-7. Chaotic solutions are omitted from these panels.

Figures 8 and 9 show two magnified examples of the two different kinds of building 
blocks in the zig-zag pattern (recognised also in Fig. 6) before and after the interaction of their $S N-P D$ bifurcation curve pairs. Figures $8 \mathrm{~A}$ and $9 \mathrm{~A}$ are the magnification of the black and purple squared domains in Fig.7. $\mathrm{D}$ at $\mu_{L}=0.100 \mathrm{Pas}$, respectively. At this viscosity value, these $S N-P D$ pairs are not connected; that is, this phase is before the interaction of the bifurcation curves and they do not form a SSD. Both Figs. $8 \mathrm{~B}$ and $9 \mathrm{~B}$ show the same bifurcation curves as panel $\mathrm{A}$ ), but at an increased dynamic viscosity value at $\mu_{L}=0.252 \mathrm{Pas}$, see also the black and purple squares in Fig. $7 \mathrm{~F}$, respectively. Here the $S N$ bifurcation curves have exchanged their subsequent $P D$ curves at the cusp point of the inner $S N$ curve, and hence, as a consequence of this interaction, two different shrimp-shaped domains are created, compare Figs. $8 \mathrm{~B}$ and $9 \mathrm{~B}$ also with Fig.11in Sec.11(Introduction).
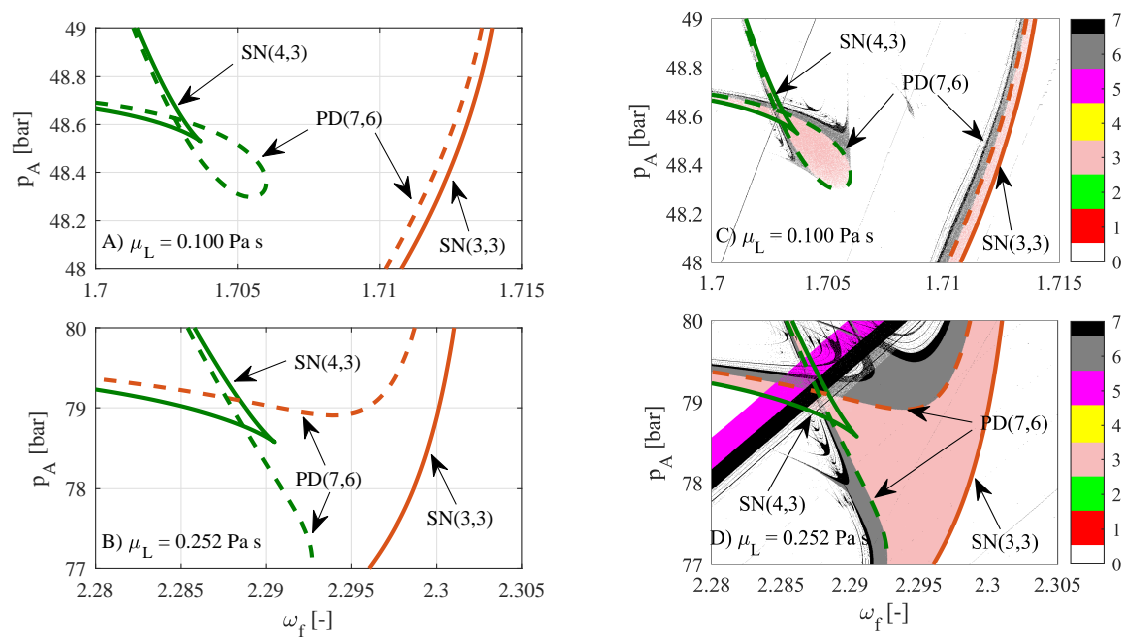

Figure 9: Resonance curves of block pattern b) creating a shrimp-shaped region as the liquid dynamic viscosity increases from $\mu_{L}=0.100 \mathrm{Pas}$ (top row) to $\mu_{L}=0.252 \mathrm{Pas}$ (bottom row). Panel A) and B) are the magnifications of the purple rectangles in Fig.7P and E, respectively. Panel C) and D) are the results of an IVP scan of the parameter areas of panel A) and B), respectively.The color code means the period of the converged periodic solutions up to period-7. Chaotic solutions are omitted from these panels.

Similarly as in case of Fig. 1. IVP scans are also provided in the right column of both figures. These diagrams show the color coded period (up to period-7) of the stable periodic solutions found. Chaotic solutions are again omitted in order to find the 
periodic structures possibly hidden by them. The period-3 and period-6 solutions are colored light pink and gray, respectively. Color white means that only chaotic solutions were found. The $S N$ and $P D$ bifurcation curves from panels A) and B) are also depicted in panels C) and D), respectively. These phase diagrams show that after the interaction of the bifurcation curves an isoperiodic domain with period-3 is created inside the domain bounded by the outer $S N$ and the two crossing $P D$ curves. According to the orders of the bifurcations curves, the two types of block patterns generate two different shrimps. This is in good accordance with the description of shrimps in the study of Medeiros et. al [14], compare Fig. 3b-c therein with the bottom panels of Figs.8 and Fig. 9

\section{The detailed mechanism of the two types of route to shrimps}

To see the mechanism behind the formation of the shrimp-shaped domains described in the previous subsection, more computations were performed with much finer increment of the dynamic viscosity $\mu_{L}$. Figure 10 shows three stages in the evolution of the bifurcation curves corresponding to block pattern a) introduced also in Fig.8. The dynamic viscosity increases from stages bottom to top, and takes the values of $0.22 \mathrm{Pas}, 0.23 \mathrm{Pas}$ and $0.24 \mathrm{Pas}$. The black curves indicate the saddle-node bifurcation curve of order $S N(4,3)$ and its first period-doubling of order $P D(7,6)$. The red and blue curves are the bifurcations of orders $S N(4,3)$ and $P D(9,6)$, respectively. Observe that the two $S N$ resonances have the same order, and they collide approximately at $\mu_{L}=0.23$ Pas indicated by the black dot in Fig. 10 At this critical point, the branches at the lower branch of the $S N$ curves are interchanged. This is clearly indicated by the fact that the blue left hand side of the red curve follows the period-doubling curve of order $P D(9,6)$ at $\mu_{L}=0.22 \mathrm{Pas}$ (lower stage), while it follows the black period-doubling curve of order $P D(7,6)$ at $\mu_{L}=0.24 \mathrm{Pas}$ (upper stage). Due to the interchange of the two $S N$ branches, the four bifurcation curves become structurally "bounded" forming the skeleton of a shrimp-shaped domain. Observe that the upper stage in Fig.10 have the same structure (although a bit deformed) as the one presented in the bottom row of 
ously deformed into the case presented in Fig. $8 \mathrm{~B}$ without the change in its topology.

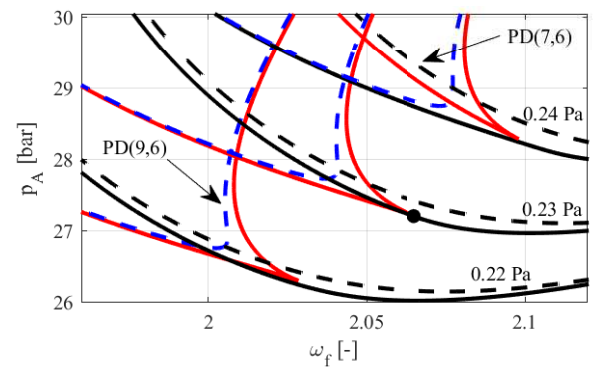

Figure 10: Formation of a shrimp-shaped domain corresponding to block pattern a) identified in Fig. 8 The dynamic viscosity increases from stages bottom to top, and takes the values of $0.22 \mathrm{Pas}, 0.23 \mathrm{Pas}$ and 0.24 Pas. Black solid and dashed curves are the $\operatorname{SN}(4,3)$ and $\operatorname{PD}(7,6)$ resonance curves, respectively. The blue dashed and the red solid curves are the $\operatorname{PD}(9,6)$ and $\mathrm{SN}(4,3)$ resonance curves, respectively.

Since block pattern a) have a significant displacement in the parameter plane with the alteration of the viscosity, Fig.10 can focus only on a narrow range of the viscosity values near the point of the interaction of the bifurcation curves. In order to obtain a global, good impression of the formation the corresponding SSD, several GPU accelerated IVP computations were performed similarly to the ones shown already in Figs. 8 C-D and Fig. 9 C-D. The viscosity was varied between $0.1 \mathrm{Pas}$ and $0.25 \mathrm{Pas}$ with an increment of $0.01 \mathrm{Pas}$. The range of the $p_{A}-\omega_{f}$ parameter plane is always adjusted to centre the interesting bifurcation structure. From the total 16 number of pictures, an animation is created, in which the approach of the two SN-PD pairs, their merge and the formation of an SSD are clearly recognisable. The animation is available as a downloadable supplementary material of the paper (RouteToShrimp.gif).

Figure 11 shows the evolution of the bifurcation curves corresponding to block pattern b) introduced also in Fig.9. The presented two stages are related to liquid dynamic viscosities $\mu_{L}=0.172$ Pas (black curves) and $\mu_{L}=0.173$ Pas (red curves). Here, the $S N$ and $P D$ curves are not distinguished via separate colour codes or line styles. In this case, the period-doubling bifurcation curves have the same order of $P D(7,6)$, which collide somewhere between the two viscosity values. The black and red curves are the states before and after the collision, respectively. Observe how the loop of one of the $P D(7,6)$ curve breaks up during the collision and interact with the other $P D(7,6)$ curve 
(the area is encircled with the light green circle). With further increase of the viscosity, the "neck" between the red curves widens and the shape of this red structure becomes more recognizable as a shrimp-shaped domain shown in Fig.9 $9 \mathrm{~B}$ and $\mathrm{D}$ (animation was not created for this case to save computational resources).

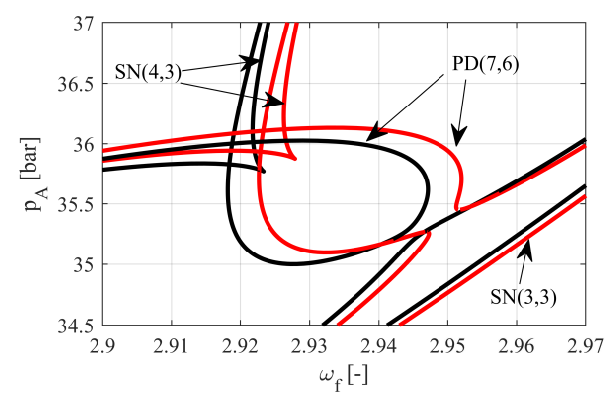

Figure 11: Formation of a shrimp-shaped domain corresponding to block pattern b) identified in Fig.9 Liquid dynamic viscosities: $\mu_{L}=0.172 \mathrm{Pas}$ (black curves) and $\mu_{L}=0.173 \mathrm{~Pa}$ (red curves). The loop of one of the $P D(7,6)$ curve breaks up during the collision and interacts with the other $P D(7,6)$ curve.

\section{Conclusion}

The subharmonic resonances of the bifurcation structure of order $(1, m)$ of the Keller-Miksis bubble oscillator was numerically investigated. In the two dimensional parameter space of the external forcing, the internal structure of the period-3 solution with order $(1,3)$ was computed by means of initial value problem solver and boundary value problem solver with numerical continuation method. This inner structure is mainly built up by pairs of saddle node $(S N)$ and period-doubling $(P D)$ bifurcation curves discussed through Figs. 5. 6 and 7. Results show that the formation of shrimp-shaped domains is highly dependent on the damping of the system that is clearly demonstarted via the supplementary material (RouteToShrimp.gif). In case of the employed model, with the parameters determined for pure water (low viscosity), the two dimensional bifurcation structure completely lacks these structures, see the upper panel of Fig.5 Increasing the damping parameter (dynamic viscosity) of the system, two mechanisms for the creation of shrimp-shaped domains were identified, see the details in Sec.6 In the first case, two $S N$ curves with the same orders collide and in- 
same orders collide and form another pair of $P D$ bifurcations. In both mechanism, two $S N-P D$ pairs interact with each other resulted in a shrimp-shaped domain.

\section{Acknowledgement}

This research was supported by the ÚNKP-16-3-I. New National Excellence Program of the Ministry of Human Capacities, by the János Bolyai Research Scholarship of the Hungarian Academy of Sciences and by the Higher Education Excellence Program of the Ministry of Human Capacities in the frame of Water science \& Disaster Prevention research area of Budapest University of Technology and Economics (BME FIKP-VÍZ).

\section{References}

[1] C. Bonatto, J. C. Garreau, J. A. C. Gallas, Self-similarities in the frequencyamplitude space of a loss-modulated $\mathrm{co}_{2}$ laser, Phys. Rev. Lett. 95 (2005) 143905.

[2] C. Scheffczyk, U. Parlitz, T. Kurz, W. Knop, W. Lauterborn, Comparison of bifurcation structures of driven dissipative nonlinear oscillators, Phys. Rev. A 43 (12) (1991) 6495-6502.

[3] D. Sornette, Why Stock Markets Crash: Critical Events in Complex Financial Systems, Princeton University Press, New Jersey, 2004.

[4] E. J. R. Parteli, M. A. F. Gomes, V. P. Brito, Nontrivial temporal scaling in a Galilean stick-slip dynamics, Phys. Rev. E 71 (3) (2005) 036137.

[5] S. H. Strogatz, Nonlinear Dynamics and Chaos with Applications to Physics, Bi355 ology, Chemistry, and Engineering, 2nd Edition, Westview Press, Boulder, Colorado, 2014.

[6] R. Varga, G. Paál, Numerical investigation of the strength of collapse of a harmonically excited bubble, Chaos Solitons Fract. 76 (2015) 56-71. 
[7] D. M. Maranhão, Ordered and isomorphic mapping of periodic structures in the

[15] J. A. de Oliveira, L. T. Montero, D. R. da Costa, J. A. Méndez-Bermúdez, R. O. Medrano-T, E. D. Leonel, An investigation of the parameter space for a family of dissipative mappings, Chaos: An Interdisciplinary Journal of Nonlinear Science 29 (5) (2019) 053114. doi:10.1063/1.5048513. 
[16] T. S. Krüger, P. C. Rech, Dynamics of an erbium-doped fiber dual-ring laser, The European Physical Journal D 66 (1).

[17] S. L. T. de Souza, A. A. Lima, I. L. Caldas, R. O. Medrano-T, Z. O. GuimaräesFilho, Self-similarities of periodic structures for a discrete model of a two-gene system, Phys. Lett. A 376 (15) (2012) 1290-1294.

[18] A. Hoff, D. T. da Silva, C. Manchein, H. A. Albuquerque, Bifurcation structures and transient chaos in a four-dimensional chua model, Physics Letters A 378 (3) (2014) $171-177$.

[19] A. Celestino, C. Manchein, H. A. Albuquerque, M. W. Beims, Stable structures in parameter space and optimal ratchet transport, Commun. Nonlinear Sci. Numer. Simul. 19 (1) (2014) 139-149.

[20] D. R. da Costa, M. Hansen, G. Guarise, R. O. Medrano-T, E. D. Leonel, The role of extreme orbits in the global organization of periodic regions in parameter space for one dimensional maps, Physics Letters A 380 (18) (2016) 1610 - 1614.

[21] C. Manchein, R. M. da Silva, M. W. Beims, Proliferation of stability in phase and parameter spaces of nonlinear systems, Chaos: An Interdisciplinary Journal of Nonlinear Science 27 (8) (2017) 081101.

[22] K. Klapcsik, R. Varga, F. Hegedús, Bi-parametric topology of subharmonics of an asymmetric bubble oscillator at high dissipation rate, Nonlinear Dynamics.

[23] E. Medeiros, S. de Souza, R. Medrano-T, I. Caldas, Replicate periodic windows in the parameter space of driven oscillators, Chaos, Solitons \& Fractals 44 (11) (2011) $982-989$.

[24] F. T. Arecchi, R. Meucci, G. Puccioni, J. Tredicce, Experimental evidence of subharmonic bifurcations, multistability, and turbulence in a $q$-switched gas laser, Phys. Rev. Lett. 49 (1982) 1217-1220.

[25] R. Vitolo, P. Glendinning, J. A. C. Gallas, Global structure of periodicity hubs in lyapunov phase diagrams of dissipative flows, Phys. Rev. E 84 (2011) 016216. 
[26] P. C. Rech, Period-adding and spiral organization of the periodicity in a hopfield neural network, International Journal of Machine Learning and Cybernetics 6 (1).

[27] R. E. Francke, T. Pöschel, J. A. C. Gallas, Zig-zag networks of self-excited periodic oscillations in a tunnel diode and a fiber-ring laser, Phys. Rev. E 87 (4) (2013) 042907.

[28] E. J. Doedel, B. E. Oldeman, A. R. Champneys, F. Dercole, T. F. Fairgrieve, Y. A. Kuznetsov, R. Paffenroth, B. Sandstede, X. Wang, C. Zhang, AUTO-07P: continuation and bifurcation software for ordinary differential equations, Concordia University, Montreal, Canada (2012).

[29] W. Façanha, B. Oldeman, L. Glass, Bifurcation structures in two-dimensional maps: The endoskeletons of shrimps, Physics Letters A 377 (18) (2013) 1264 1268.

[30] J. B. Keller, M. Miksis, Bubble oscillations of large amplitude, J. Acoust. Soc. Am. 68 (2) (1980) 628-633.

[31] W. Lauterborn, T. Kurz, Physics of bubble oscillations, Rep. Prog. Phys. 73 (10) (2010) 106501.

[32] F. Hegedús, K. Klapcsik, The effect of high viscosity on the collapse-like chaotic and regular periodic oscillations of a harmonically excited gas bubble, Ultrason. Sonochem. 27 (2015) 153-164.

[33] C. E. Brennen, Cavitation and Bubble Dynamics, Oxford University Press, New York, 1995.

[34] E. KLASEBOER, S. W. FONG, C. K. TURANGAN, B. C. KHOO, A. J. SZERI, M. L. CALVISI, G. N. SANKIN, P. ZHONG, Interaction of lithotripter shockwaves with single inertial cavitation bubbles, Journal of Fluid Mechanics 593 (2007) 33-56. doi:10.1017/S002211200700852X.

[35] Y. Zhang, Y. Zhang, Z. Qian, B. Ji, Y. Wu, A review of microscopic interactions between cavitation bubbles and particles in silt-laden flow, Renewable and Sus- 
tainable Energy Reviews 56 (2016) 303-318. doi:10.1016/j.rser.2015.11.

[36] Y. Zhang, Y. Zhang, Chaotic oscillations of gas bubbles under dual-frequency acoustic excitation, Ultrasonics Sonochemistry 40 (2018) 151 - 157, sI: ESS-15, 2016, Istanbul.

[37] Y. Zhang, Z. Guo, X. Du, Wave propagation in liquids with oscillating vaporgas bubbles, Applied Thermal Engineering 133 (January) (2018) 483-492. doi: 10.1016/j.applthermaleng.2018.01.056

[38] Y. Zhang, F. Chen, Y. Zhang, Y. Zhang, X. Du, Experimental investigations of interactions between a laser-induced cavitation bubble and a spherical particle, Experimental Thermal and Fluid Science 98 (June) (2018) 645-661. doi:10. 1016/j.expthermflusci.2018.06.014

[39] H. Haghi, A. Jafari Sojahrood, R. Karshafian, M. C. Kolios, Numerical investigation of the subharmonic response of a cloud of interacting microbubbles, The Journal of the Acoustical Society of America 141 (5) (2017) 3493-3493. doi:10.1121/1.4987295.

[40] H. Haghi, A. Jafari Sojahrood, M. C. Kolios, On amplification of radial oscillations of microbubbles due to bubble-bubble interaction in polydisperse microbubble clusters under ultrasound excitation, The Journal of the Acoustical Society of America 143 (3) (2018) 1862-1862.

[41] H. Haghi, A. Jafari Sojahrood, A. C. De Leon, A. Exner, M. C. Kolios, Experimental and numerical investigation of backscattered signal strength from different concentrations of nanobubble and microbubble clusters, The Journal of the Acoustical Society of America 144 (3) (2018) 1888-1888. doi:10.1121/1. 5068280

[42] U. Parlitz, W. Lauterborn, Resonances and torsion numbers of driven dissipative nonlinear oscillators, Z. Naturforsch. A 41 (4) (1986) 605-614. 
[43] L. Haar, J. S. Gallagher, G. S. Kell, NBS/NRC Wasserdampftafeln, Springer, Berlin, 1988.

[44] C. Westbrook, Calculate density and viscosity of glycerol/water mixtures (2018).

11 URL http://www.met.reading.ac.uk/ sws04cdw/viscosity_calc. html

[45] K. E. Niemeyer, C.-J. Sung, Accelerating moderately stiff chemical kinetics in reactive-flow simulations using gpus, Journal of Computational Physics 256 (2014) 854-871.

[46] P. Beiersdorfer, J. M. Wersinger, Y. Treve, Topology of the invariant manifolds of period-doubling attractors for some forced nonlinear oscillators, Phys. Lett. A 96 (6) (1983) 269-272.

[47] P. Beiersdorfer, Universality of the topology of period doubling dynamical systems, Phys. Lett. A 100 (8) (1984) 379-382.

[48] R. Gilmore, Topological analysis of chaotic dynamical systems, Rev. Mod. Phys. 70 (4) (1998) 1455-1529.

[49] F. Hegedûs, C. Hôs, L. Kullmann, Stable period 1, 2 and 3 structures of the harmonically excited Rayleigh-Plesset equation applying low ambient pressure, IMA J. Appl. Math. 78 (6) (2013) 1179-1195.

[50] F. Hegedús, Stable bubble oscillations beyond Blake's critical threshold, Ultrasonics 54 (4) (2014) 1113-1121.

[51] C. J. Hôs, A. R. Champneys, K. Paul, M. McNeely, Dynamic behaviour of direct spring loaded pressure relief valves in gas service: II reduced order modelling, J. Loss Prevent. Proc. 36 (2015) 1-12.

[52] M. M. Fyrillas, A. J. Szeri, Dissolution or growth of soluble spherical oscillating bubbles, J. Fluid Mech. 277 (1994) 381-407. 
[53] W. Knop, W. Lauterborn, Bifurcation structure of the classical morse oscillator,

n The Journal of Chemical Physics 93 (6) (1990) 3950-3957. doi:10.1063/1. 458780

[54] V. Englisch, W. Lauterborn, Regular window structure of a double-well duffing oscillator, Phys. Rev. A 44 (1991) 916-924. doi:10.1103/PhysRevA.44.916 